\title{
A virtual reality simulator combining a learning environment and clinical case database for image-guided prostate biopsy
}

\author{
Sonia-Yuki Selmi ${ }^{1}$, Gaelle Fiard ${ }^{2,1}$, Emmanuel Promayon ${ }^{1}$, Lucile Vadcard ${ }^{3}$, Jocelyne Troccaz $^{1,2}$ \\ ${ }^{1}$ UJF-Grenoble 1 / CNRS / TIMC-IMAG UMR 5525, Grenoble, F-38041, France \\ ${ }^{2}$ Grenoble University Hospital, Department of Urology, Grenoble, France \\ ${ }^{3}$ LSE, UPMF, Grenoble, France \\ FirstName.Name@imag.fr
}

\begin{abstract}
The recent availability of navigation systems for mapping and targeting of transrectal ultrasound (TRUS) guided prostate biopsies revealed new opportunities in training the clinician. This paper describes a simulator for TRUS guided prostate biopsy that offers similar information, enhanced by a complete learning environment. Various exercises have been developed in accordance with a didactical study identifying the training needs. A dedicated clinical case database fed by a prostate navigation system provides a large patient prostate image database that covers the main situations encountered during clinical practice. A haptic device is used to enable complete biopsy procedures or practice specific tasks. This paper also presents work in progress of the evaluation of such a simulator.
\end{abstract}

\section{Introduction}

Virtual reality surgical simulators, as educational tools, allow medical practitioners to perform complex procedures in a controlled environment and provide an independent evaluation system [1].

Prostate cancer is the second most common cancer worldwide for males [2]. Prostate biopsy procedures, performed to obtain and analyze tissue samples of the gland, are required for diagnosis confirmation. Conventional biopsies are performed under transrectal ultrasound (TRUS) guidance (see fig. 1) and the initial training, performed through an apprenticeship, can be challenging for the trainee, painful for the patient, and does not allow quantitative evaluation. Indeed, the exact position of the biopsy is not known.

\subsection{Context}

The quality of a prostate biopsy procedure is essential for the initial diagnosis and management of prostate cancer. Current teaching methods, based on apprenticeship without feedback on biopsy distribution and overall performance led to a low correlation between the presumed biopsy location and the final pathology of the prostatectomy specimen [3]. Previous work showed that a visual feedback about the real location of performed biopsy sessions allowed improvement of the biopsy distribution, even for an experienced operator [4].

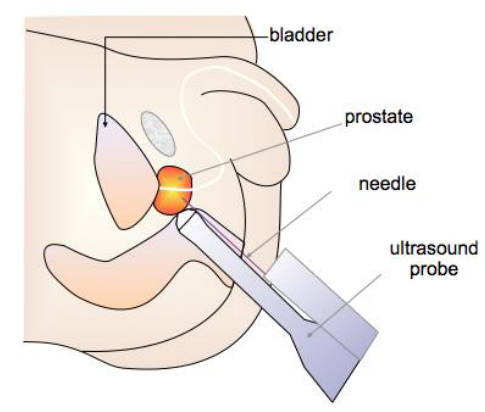

Figure 1. Anatomy of the prostate and imaging by transrectal ultrasound

The prostate biopsy procedure is challenging for several reasons. First of all, it requires mental reconstruction of a three dimensional (3D) gland (almost spherical, $4 \mathrm{~cm}$ in diameter) based on twodimensional (2D) TRUS images, and a good hand-eye coordination to regularly distribute biopsies in order to sample each part of the gland, as previous biopsies are not displayed on the US image. Secondly, tasks vary from randomized sampling to very precise targeting, which becomes necessary with the emergence of targeted biopsies on MRI suspicious areas. Also, the prostate moves and is deformed due to the ultrasound probe movements during a biopsy session. Moreover, patient anatomy varies and the gesture must be adapted to various prostate sizes. Finally, except the display of 
the current 2D US image where a biopsy occurs, no visual feedback or evaluation of the biopsy location in the 3D prostate volume can be obtained from a conventional biopsy procedure. Besides, recent progress in Magnetic Resonance Imaging (MRI) has allowed the definition of suspicious areas inside the prostate prior to biopsy, and the performance of targeted biopsies on these areas. Targeting under US imaging an area detected on another imaging modality (i.e., MRI) can be performed using mental fusion or navigation systems, and there is a need for simulation of this specific task.

A recent report by the French Health Authorities (HAS) revealed an insufficient use and a lack of validated surgical simulators, although specifications and conditions of use have been well specified by a meta-analysis [5][1]. These conditions of use include a learning environment around the simulator, as a hyperrealistic simulator does not guarantee its educational interest [6]. Explanations and guidance of the trainee are necessary to take the full benefit of simulations and facilitate the transfer of the acquired skills to biopsy real patients. Besides, a balance between repetitive practice and experience of varying situations is essential for performance improvement.

\subsection{State of the art}

Different types of simulators have been developed to teach and improve technical and non-technical medical skills. Surgical simulators represent a small proportion of available simulators, and most of them are dedicated to laparoscopic skills [5]. In the field of urology, simulators of robotic, laparoscopic and endoscopic procedures have been described or commercialized [7][8]. Some of them have demonstrated reliability (reproducibility for the same user), face validity (realism of the simulator assessed by nonexperts), content validity (assessed by experts, the simulator teaches what it is supposed to teach) and construct validity (the simulator is able to discriminate between novices and experts), but not all showed a clinical benefit (i.e., the ability to transpose acquired skills in clinical practice)[9].

To our knowledge, mainly two devices were designed for the simulation of prostate biopsy procedures. Xang et al. [10] and Zeng et al. [11] developed 3D simulation models but used it to evaluate the efficiency of various biopsy schemes rather than for educational purposes. Chalasani et al. [12] reported the development and face, content and construct validation of a Virtual Reality TRUS guided prostate biopsy simulator, which allowed the performance of virtual conventional prostate biopsies with assistance, with a limited teaching environment.

This paper presents the design of a complete virtual reality learning environment for prostate biopsy teaching, practicing and evaluation, based on real patient cases and containing a set of exercises allowing for personalized learning paths. The next sections describe the simulator and the planned modalities of its validation.

\section{Methods and tools}

\subsection{Training needs assessment}

In order to obtain a real clinical benefit, a simulator has to help the students not only to learn the gesture, but also to learn and train on the prostatic biopsy procedure as a whole. In order to ensure this, the simulator has to integrate multiple functions: make the link between theory and practice, offer exercises which target different aspects of prostate biopsy, contain various patient cases and provide increasing difficulty for biopsy.

A didactical study was performed to evaluate the training needs [13]. Its purpose was to identify the activities of trainees and to analyze how reference situations can be transposed into a simulator. It allowed the development of specific exercises, taking into account individualized learning pathways.

A biopsy procedure requires understanding handeye coordination using both the 2D TRUS image and the virtual probe. The aim is to develop jointly skills and the good 3D mental representation they require. Pre-requisites include ultrasound image reading, prostate volume measurement and ability to determine the probability of finding a cancer based on clinical data (prostate specific antigen (PSA), prostate volume, digital rectal examination (DRE)).

In clinical practice, a 12-core biopsy protocol is usually performed, using an end-fire probe. The probe is held in one hand and the other hand is needed to fire the biopsy. The patient can be installed in either dorsal or lateral decubitus position, and biopsies can be performed using 2D axial or transversal US view.

The gland is divided in 12 areas usually called quadrants (see fig. 2) and clinicians take sample in each quadrant of the prostate in order to obtain welldistributed biopsy samples. Clinicians generally start to perform the biopsy on one side of the prostate (starting by the base or the apex) and take six samples. Then, they do the other side symmetrically. The order of biopsy depends on clinicians' habits. Learners have to learn how to biopsy a target or a "quadrant" of the prostate. Performing biopsy with assistance (3D 
representation of the US plane and the prostate) can help users build a 3D mental representation.

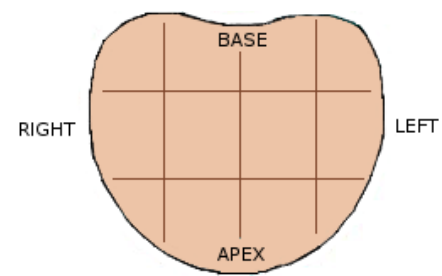

Figure 2. Prostate scheme and the twelve quadrants (transverse view)

\subsection{Biopsym simulator}

The Biopsym simulator is a complete learning environment for prostate biopsy. Based on a first version of the simulator [14][15], the second version presented here is connected to a clinical database where patient cases can be added (see 4.1.2) and provides a complete realistic virtual environment, where the previously identified relevant for learning conditions of a prostate biopsy procedure are replicated.

2.2.1. System architecture. The simulator (see fig. 3 ) is running on a laptop computer connected, on one hand, to a haptic interface Phantom Omni (Sensable Devices Inc., MA, USA) and, on another hand, to a CamiTK-based graphical user interface software [16]. CamiTK ${ }^{1}$ is an open-source modular framework, developed to prototype Computer Assisted Medical Interventions applications. CamiTK viewers are used for data insertion and biopsy feedback. The simulator also uses Insight and Registration Toolkit (ITK) and Visualization Toolkit (VTK) libraries for data processing and representations. The interface is developed with Qt, which allows an intuitive manipulation of the software.

We use the OpenHaptic ${ }^{\mathrm{TM}}$ library provided by Sensable Devices Inc. to handle communication with the Phantom. The low-level library gives access to the haptic device configuration, which allows the simulator, for instance, to get the device position and orientation. The high-level library is used for haptic rendering, in order to provide force effects.

\subsubsection{Databases.}

Medical data. Patient data are collected and anonymized from the UroStation $®$ system (Koelis, France). This system gives us access to a complete database containing 3D US prostate volumes, MRI exams, prostate meshes and image fusion information that enable the registration of all these data in a single

\footnotetext{
${ }^{1}$ See http://camitk.imag.fr
}

reference system. All TRUS images are acquired during a prostate biopsy procedure on patients who are suspected of cancer. Ultrasound image is acquired from an end-fire 3D TRUS probe.

Each patient case in the database is composed of clinical information (PSA, prostate volume, DRE and age), an ultrasound image (including a targeted area), an MRI image (including a targeted area) and a prostate mesh. After a process of data anonymization, a dozen patient cases were integrated into the simulator to offer a good sample of what can be expected in clinical practice. The database is not limited and new cases can be easily added through an administrator access.

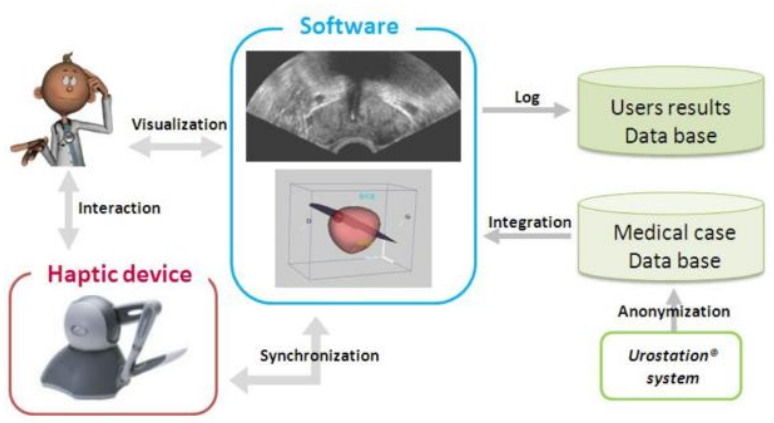

Figure 3. Simulator architecture

User data. The user database contains information about the simulator users and records their biopsy results and exercise results. It allows users to access their personal performance (score, minimum and maximum score, average time, cumulated time and so on...). The purpose of this part of the database is to provide the users an overview of their improvement and trace of the training process. Extraction of these data will also allow statistical analysis to facilitate the evaluation of the simulator itself. Both databases are managed by the open source relational database management system MySQL (Oracle, CA, USA).

2.2.3. Simulator usage. The haptic device is used, as a motion tracker, the device stylus representing the virtual US probe (see fig. 4). The Phantom device provides a constant force to replicate the tissue friction.

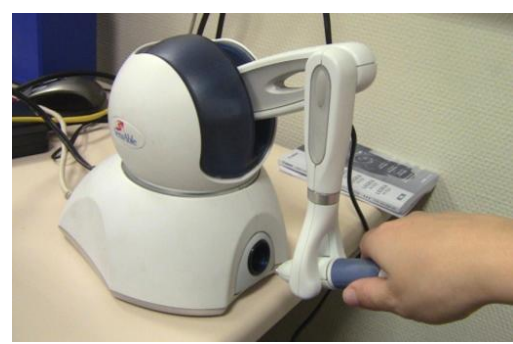

Figure 4. Omni Phantom device 
The 2D TRUS image plane is displayed in realtime in function of the virtual probe orientation. Guided by the 2D TRUS image plane, the users move the probe to perform the biopsy. As in a real biopsy procedure, the needle guide is fixed on the US probe, the virtual guide position is defined directly from the stylus position and orientation. The fixed guide position displays the potential trajectory of the needle on the 2D TRUS image plane. Learners have to use the laptop's keyboard to adapt the needle length and take a sample. The buttons on the Phantom stylus are not used.

2.2.4. Exercises. According to the previously led didactical study, we implemented various exercises, which allow each to develop an aspect of the required skills.

Specific exercises. Seven exercises have already been implemented to target different aspects of the surgical gesture and help understand hand-eye coordination using both the two-dimensional (2D) TRUS image and the virtual probe.

The first two exercises test basic knowledge about US image reading by asking the user to select the different anatomical structures on the image.

The third exercise also evaluates the US image knowledge and corresponding 3D representation by asking the user to measure the prostate volume.

The fourth exercise tests more fundamental urology knowledge by asking the user an estimation of the probability of positive biopsies based on clinical data.

Three other exercises are dedicated to targeted biopsy. Learners have to biopsy a target or a specific "quadrant" of the prostate. The target is previously defined during data integration in the database. One target is placed on the ultrasound image and another one is placed on the MR image. The targets are then displayed in the virtual 3D representation using MRUS image registration.

Each of these three exercises is divided into two levels of increasing difficulty. In the easier level, users perform the biopsy with assistance; it means that, in addition to the usual 2D TRUS image, a 3D representation of the prostate with the current probe plane position is displayed (see fig. 5). The 3D representation displays the prostate mesh as well as the position of the TRUS image slice. This representation helps to build a correct visualization of the TRUS image relatively to the prostate (see fig. 6).

Each exercise provides the users with a score representing their performance.

Virtual biopsy procedure. Finally, the simulator offers the ability to perform an entire virtual biopsy procedure. This exercise requires all the skills acquired during the various specific exercises and completely mimics the actual procedure performed clinically on patients.

The simulator can be configured to perform the biopsy with the patient either in decubitus position or in lateral position. Moreover, the initial view can be either the axial view or the sagittal view. Finally, biopsy order is chosen by the user at the beginning of the simulation (with the choice of a procedure starting at the prostate left central base, left lateral base, right central base, or right lateral base).

To improve the realism and educational interest, learners have to complete a pre-operative checklist before beginning the procedure, based on the checklist used in clinical practice. The assistance of the 3D visualization can also be provided in this exercise.

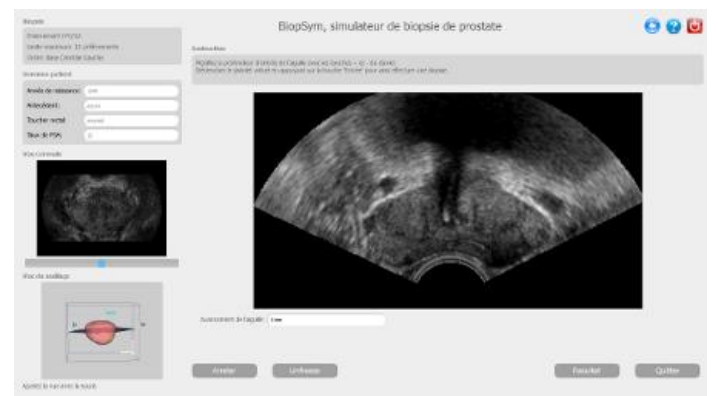

Figure 5. Biopsy control screen with the 2D TRUS plane (main frame), a coronal view and a 3D representation of the prostate (on the left)

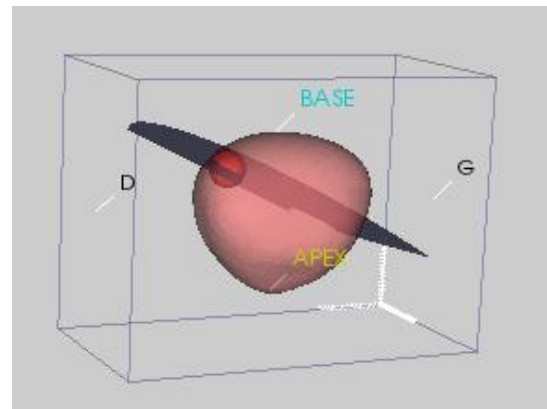

Figure 6. The 3D representation of the prostate inside the TRUS volume box and the current position of the probe plane. For targeted biopsy, the target is visible.

\section{Results}

The simulator we developed provides a complete learning environment and allows users to be evaluated.

The current state of the simulator can be visualized at http://www.youtube.com/watch?v=FeTtc5d_kQc (virtual assisted biopsy procedure) and http://www.youtube.com/watch? $\mathrm{v}=\mathrm{q} 5 \mathrm{q} 1 \mathrm{JUPbVmE}$ (exercise with targeted biopsy). 


\subsection{Performance assessment}

Once the learners have completed their biopsies, they can access their results by visualizing sample positions relatively to the $3 \mathrm{D}$ image of the prostate in a CamiTK 3D interactive viewer (see fig. 7). This visualization offers a precise feedback about the biopsy. For each sample, learners can access to the following information: (1) sample location in the ultrasound image and prostate mesh (biopsy mapping) (2) sample position relatively to the targeted quadrant (3) sample length inside the targeted quadrant. The success rate (given as the percentage of the sample positions in their targeted quadrants) is given.

The simulator also provides the biopsy duration and the number of non-biopsied quadrants. This allows the score to also take into account the duration of the procedure relatively to a reference duration as well as a coverage rate.

Using this information, we can evaluate the quality of the biopsy. The biopsy results are also saved in the same reference frame as the patient images, mesh and biopsy location. CamiTK facilitates the analysis of the results in order to assess the impacts of the simulator on physician training. The scoring system could be defined as a combination of precision, missed area, and duration relatively to simulations performed by experts and in collaboration with educational scientists.

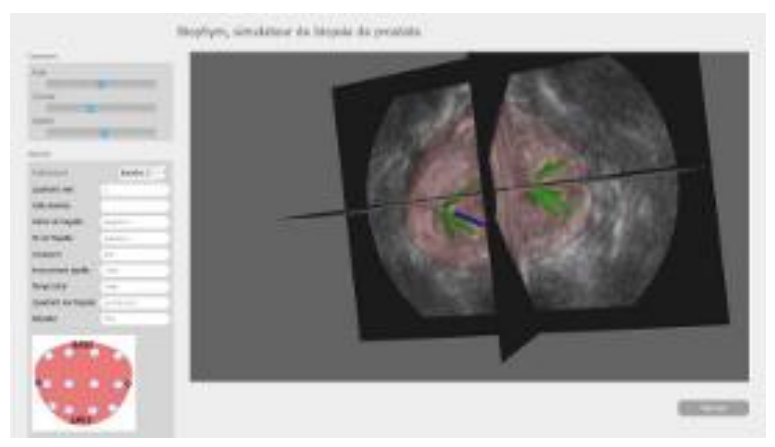

Figure 7. Result visualization

\subsection{Evaluation}

The first exploratory evaluation was performed to obtain feedback on the software and the haptic device from a first set of users, and to demonstrate face validity. Eight non-clinicians $(\mathrm{PhD}$ and Masters students, researchers in computer assisted medical interventions) performed a virtual biopsy session with the simulator and filled an evaluation questionnaire. Six of them thought the simulation was close to what they imagined; the other two did not give an opinion. All of them thought the simulator was easy to use and intuitive, and thought the scoring of the performance was important.

The next step consisted in the evaluation of the simulator by clinicians. Novices (medical students) and experts (trained urologists) looked through the various exercises and educational contents and performed virtual biopsies on the simulator. Experts validated the content and suggested improvements notably in the realism of the ultrasound probe. The basic scoring system used did not significantly discriminate between a limited sample $(\mathrm{n}=21)$ of novices and experts and will have to be improved and validated on a larger population.

\section{Discussion}

As mentioned before, our aim is to reach the exact level of realism that serves our training goals. Thereby, more realism could be achieved by including prostate deformations due to the probe. A model of prostate deformation under TRUS examination would have to be developed in order to compute in real-time the prostate movements and distortions. Whether this could improve the simulation realism would also have to be evaluated.

We chose to use the keyboard to fire the virtual needle to force the use of both hands, as it is the case during a real procedure.

The different exercises have been implemented. A decision-making system which can automatically provide the best exercise depending on previous results of a given user has been described [13]. It would provide the creation of personalized learning paths and also has to be implemented.

To evaluate the educational interest, an evaluation is scheduled in 2013 among a population of novices (medical students), in order to compare the learning of the prostate biopsy procedure with or without the use of the simulator.

\section{Conclusion}

In this paper, we have described a complete training simulator for TRUS prostate biopsy. In a realistic virtual environment, learners can perform the complete prostate biopsy procedure.

We have worked on didactical aspects of the simulator to analyze training needs and develop exercises targeting multiple tasks of the gesture.

A complete evaluation is scheduled to attest for the clinical interest of our simulator.

The development of this simulator meets the training needs for augmented-surgery systems such as the Urostation ${ }^{\circledR}$, which provide access to new data, 
especially the fusion between medical imaging modalities. Besides, virtual reality based simulators give the clinicians a first experience of robotic systems and computer-assisted medical systems.

The evaluation of the educational value of the simulator and the transfer of the acquired skills to an actual patient would conclude the validation of the simulator, with the aim of obtaining a reliable tool for initial training, evaluation and certification, for conventional randomized biopsies or targeted biopsies with assistance.

\section{Acknowledgments}

This work was supported by French state funds managed by the ANR within the Investissements d'Avenir programme (Labex CAMI) under reference ANR-11-LABX-0004, the Association Française d'Urologie - AstraZeneca (grant G.Fiard) and by INSERM CHRT (grant J.Troccaz). We want to thank Dr Pierre Mozer, from La Pitié Salpétrière Hospital, Paris, for his strong involvement in the early versions of Biopsym and Koelis for giving us access to internal data structures of the Urostation ${ }^{\circledR}$.

\section{References}

[1] S. B. Issenberg, W. C. McGaghie, E. R. Petrusa, D. Lee Gordon, and R. J. Scalese, «Features and uses of highfidelity medical simulations that lead to effective learning: a BEME systematic review », Med Teach, vol. 27, $\mathrm{n}^{\circ}$ 1, pp. 10-28, 2005.

[2] P. D. Baade, D. R. Youlden, and L. J. Krnjacki, «International epidemiology of prostate cancer: geographical distribution and secular trends », Mol Nutr Food Res, vol. 53, $\mathrm{n}^{\mathrm{o}} 2$, pp. 171- 184, 2009.

[3] V. Iremashvili, L. Pelaez, M. Jorda, M. Manoharan, M. Arianayagam, D. L. Rosenberg, and M. S. Soloway, «Prostate sampling by 12-core biopsy: comparison of the biopsy results with tumor location in prostatectomy specimens », Urology, vol. 79, n 1, pp. 37- 42, 2012.

[4] P. Mozer, M. Baumann, G. Chevreau, A. Moreau-Gaudry, S. Bart, R. Renard-Penna, E. Comperat, P. Conort, M.-O. Bitker, E. Chartier-Kastler, F. Richard, and J. Troccaz, «Mapping of transrectal ultrasonographic prostate biopsies: quality control and learning curve assessment by image processing », J Ultrasound Med, vol. 28, n 4, pp. 455- 460, 2009.

[5] Haute Autorité de Santé, « Rapport de mission. Etat de l'art (national et international) en matière de pratiques de simulation dans le domaine de la santé. » HAS, 2012 (in French).
[6] G. Norman, K. Dore, and L. Grierson, « The minimal relationship between simulation fidelity and transfer of learning », Med Educ, vol. 46, n ${ }^{\circ}$ 7, p. 636- 647, 2012.

[7] M. S. Michel, T. Knoll, K. U. Köhrmann, and P. Alken, «The URO Mentor: development and evaluation of a new computer-based interactive training system for virtual lifelike simulation of diagnostic and therapeutic endourological procedures », BJU Int., vol. 89, n 3, pp. 174- 177, 2002.

[8] H. Abboudi, M. S. Khan, O. Aboumarzouk, K. A. Guru, B. Challacombe, P. Dasgupta, and K. Ahmed, « Current status of validation for robotic surgery simulators - a systematic review », BJU Int., vol. 111, n ${ }^{\circ} 2$, pp. 194- 205, 2013.

[9] E. M. McDougall, « Validation of surgical simulators », J. Endourol., vol. 21, nº 3, pp. 244- 247, 2007.

[10] J. Xuan, Y. Wang, I. A. Sesterhenn, J. W. Moul, and S. K. Mun, «3D model supported prostate biopsy simulation and evaluation », Notes Comput Sci, vol. 1496, pp. 358- 367, 1998.

[11] J. Zeng, J. Bauer, W. Zhang, I. Sesterhenn, R. Connelly, J. Lynch, J. Moul, and S. K. Mun, « Prostate biopsy protocols: 3D visualization-based evaluation and clinical correlation », Comput. Aided Surg., vol. 6, n ${ }^{\circ}$, pp. 14- 21, 2001.

[12] V. Chalasani, D. W. Cool, S. Sherebrin, A. Fenster, J. Chin, and J. I. Izawa, « Development and validation of a virtual reality transrectal ultrasound guided prostatic biopsy simulator », Can Urol Assoc J, vol. 5, n 1, pp. 19- 26, 2011.

[13] C. Meurger, «Caractérisation didactique d'un simulateur pour la formation médico-chirurgicale », Pierre Mendes France University, Grenoble, Master Thesis, 2009 (in French).

[14] S. Sclaverano, G. Chevreau, L. Vadcard, P. Mozer, and J. Troccaz, « BiopSym: a simulator for enhanced learning of ultrasound-guided prostate biopsy », Stud Health Technol Inform, vol. 142, pp. 301- 306, 2009.

[15] T. Janssoone, G. Chevreau, L. Vadcard, P. Mozer, and J. Troccaz, « Biopsym: a learning environment for trans-rectal ultrasound guided prostate biopsies », Stud Health Technol Inform, vol. 163, pp. 242- 246, 2011.

[16] C. Fouard, A. Deram, Y. Keraval, et E. Promayon, "CamiTK: A Modular Framework Integrating Visualization, Image Processing and Biomechanical Modeling », in Soft Tissue Biomechanical Modeling for Computer Assisted Surgery, Y. Payan, Éd. Springer Berlin Heidelberg, pp. 323- 354, 2012.

11. Copyright forms and reprint orders 